\title{
A Case of Vogt-Koyanagi-Harada Disease-Like Uveitis Induced by Nivolumab and Ipilimumab Combination Therapy
}

\author{
Keisuke Minami Mariko Egawa Keisuke Kajita Fumiko Murao \\ Yoshinori Mitamura \\ Department of Ophthalmology, Institute of Biomedical Sciences, Tokushima University \\ Graduate School, Tokushima, Japan
}

\begin{abstract}
Keywords
Vogt-Koyanagi-Harada disease-like uveitis · Nivolumab · Ipilimumab · Malignant melanoma · Panuveitis
\end{abstract}

\begin{abstract}
Nivolumab and ipilimumab are widely used immune checkpoint inhibitors (ICPIs) for the treatment of metastatic melanoma. ICPIs cause an array of side effects called immune-related adverse events (IRAEs) due to activation of an immune response. ICPI-uveitis can cause irreversible vision loss if untreated. There are few reports of recurrent Vogt-Koyanagi-Harada (VKH) disease-like uveitis induced by nivolumab and ipilimumab. We report a case of VKH diseaselike uveitis recurrence after resuming ICPIs. A 73-year-old man with advanced melanoma was referred to our clinic with visual loss 25 days after starting nivolumab/ipilimumab. His corrected visual acuity was 0.5 in the right eye and 0.02 in the left eye. Enhanced-depth imaging optical coherence tomography (EDI-OCT) showed marked choroid thickening. The patient was diagnosed with VKH disease-like uveitis due to IRAEs. Subtenon injection of triamcinolone acetonide was performed, and nivolumab/ipilimumab was suspended, but serous retinal detachment (SRD) markedly worsened and choroidal detachment appeared. With 2 courses of steroid pulse therapy and oral steroids, SRD disappeared, and corrected visual acuity recovered in both eyes. Five months after the first injection, exacerbation of melanoma was observed, and nivolumab and oral steroids were restarted. Six weeks later, an increase in choroidal thickness was observed with EDI-OCT and diagnosed as a recurrence of VKH disease-like
\end{abstract}


uveitis. Monitoring for the recurrence of VKH disease-like uveitis during the administration of ICPIs, even after uveitis is treated, is essential. Assessment of choroidal thickness with EDI-OCT may be useful for detecting early signs of VKH disease-like uveitis.

(C) 2021 The Author(s).

Published by S. Karger AG, Basel

\section{Introduction}

In recent years, immune checkpoint inhibitors (ICPIs) targeting checkpoints, such as programmed cell death-1 (PD-1) and cytotoxic T-lymphocyte antigen-4 (CTLA-4), have been widely used for the treatment of metastatic carcinomas and other solid tumors. The immune system contains molecules that activate and suppress the immune response, which suppress inappropriate autoimmune and excessive inflammatory responses. CTLA-4 and PD-1 are typical immunosuppressive molecules, with CTLA-4 suppressing T-cell activation during the initial response to antigens and PD-1 suppressing peripheral tissue damage by suppressing activated T cells. ICPIs block each suppressive signal to stop the suppression of the immune system or to enhance the immune response to the tumor [1]. In recent years, nivolumab (antiPD-1 antibody) plus ipilimumab (anti-CTLA-4 antibody) have been used for the treatment of advanced melanoma [2]. While it has a highly effective therapeutic outcome, ICPIs cause a wide array of systemic side effects called immune-related adverse events (IRAEs) due to activation of an immune reaction. Recently, several case reports of Vogt-Koyanagi-Harada (VKH) disease-like uveitis caused with ICPIs have been published; however, there are few case reports of VKH disease-like uveitis recurrence after resuming ICPIs [3, 4]. We report a case in which choroidal thickness (CT) measurement by enhanced-depth imaging optical coherence tomography (EDI-OCT) was useful for evaluation of VKH disease-like uveitis relapse caused by nivolumab plus ipilimumab.

\section{Case Presentation}

A 73-year-old man was diagnosed with metastatic melanoma and received the first dose of nivolumab plus ipilimumab. Twenty-five days after injection, he was referred to our clinic with complaints of visual impairment and floaters in both eyes. There were no symptoms, such as headache or tinnitus. His best-corrected visual acuity was $0.5 \mathrm{in}$ the right eye and $0.02 \mathrm{in}$ the left eye. Intraocular pressure was $13 \mathrm{~mm} \mathrm{Hg}$ in both eyes. Moderate granulomatous anterior uveitis, swelling and hyperemia of the optic nerve head, and multiple serous retinal detachments (SRD) were found in both eyes (shown in Fig. 1a, b). Using fluorescein angiography, multiple hyperfluorescent spots, late leakage and pooling, and optic disk hyperfluorescence were found in both eyes (shown in Fig. 1c, d). Indocyanine-green angiography revealed findings characteristic of VKH disease, such as dark background during the early phase and multiple hypofluorescent dark spots during the late phase (shown in Fig. 1e, f). EDI-OCT confirmed a significant increase in CT, undulations of the retinal pigment epithelium, and SRD with the septum (shown in Fig. 2a, b). Human leukocyte antigen (HLA) typing revealed serotype group DR4.

Based on clinical findings and treatment history, we diagnosed VKH disease-like uveitis due to the adverse effect of ICPI. The second dose of nivolumab plus ipilimumab was discontinued, and we administered a bilateral subtenon injection of triamcinolone (STTA) and topical corticosteroids (betamethasone sodium phosphate $0.1 \%$ ) 4 times a day. The SRD of both eyes was found to have decreased 3 days after STTA administration; the best-corrected visual

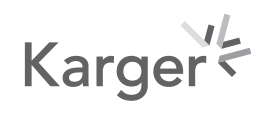




\section{Case Reports in Ophthalmology}
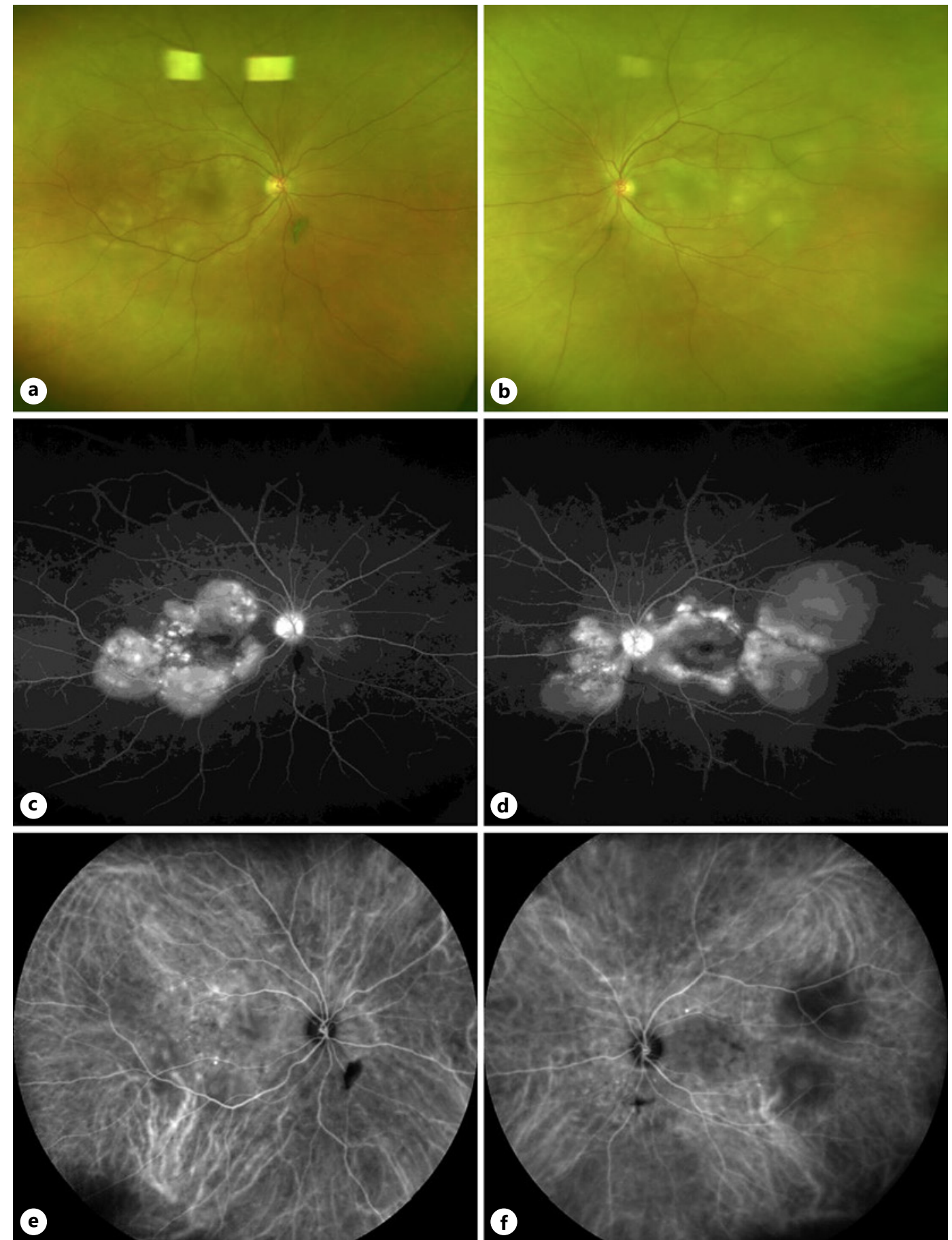

Fig. 1. Fundus photographs, fluorescein angiographic images, and indocyanine-green angiographic images before treatment. Left column shows the right eye, and the right column shows the left eye. a, b Fundus photographs show multiple sites of serous retinal detachment and subretinal deposition of yellowish-white fibrinlike material in both eyes. $\mathbf{c}, \mathbf{d}$ Fluorescein angiographic images show pooling corresponding to multiple serous retinal detachments and optic disk hyperfluorescence in the late phase. e, $\mathbf{f}$ Indocyanine-green angiographic images show multiple hypofluorescent dark spots in the intermediate to late phase. 


\section{Case Reports in Ophthalmology}
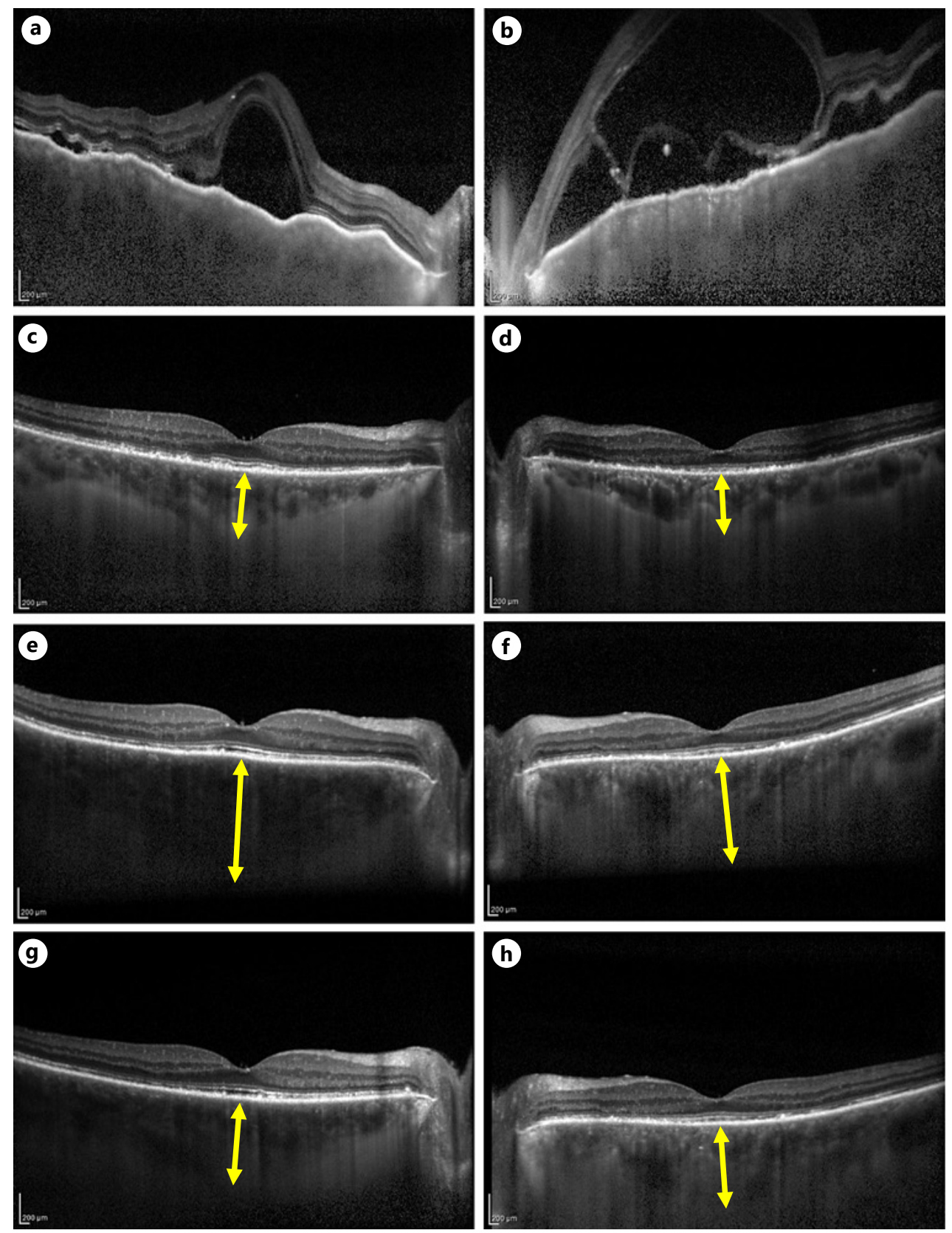

Fig. 2. EDI-OCT images during patient follow-up. Left column shows EDI-OCT images of the right eye, and the right column shows those of the left eye. a, b Before treatment, SRD, choroidal folds, and marked choroidal thickening are observed (CT cannot be measured). c, $\mathbf{d}$ After the second steroid pulse treatment, serous retinal detachment disappeared and CT decreased (CT: right eye $390 \mu \mathrm{m}$, left eye $330 \mu \mathrm{m})$. e, f Nivolumab administration was resumed, and there was no significant change in the first administration, but CT increased and inflammation recurred in the second administration (CT: right eye $665 \mu \mathrm{m}$, left eye $485 \mu \mathrm{m}$ ). $\mathbf{g}$, h One week after STTA was performed on both eyes for relapse. Choroidal thickening in both eyes improved. (CT: right eye $370 \mu \mathrm{m}$, left eye $350 \mu \mathrm{m}$ ). EDI-OCT, enhanced-depth imaging optical coherence tomographic; CT, choroidal thickness; STTA, subtenon injection of triamcinolone acetonide.

acuity improved to 0.2 in the left eye. However, 10 days later, a marked exacerbation of SRD and choroidal detachment was observed, and the corrected visual acuity dropped to 0.15 in the right eye and 0.1 in the left eye. On the twelfth day following the first visit, we started a 
methyl-prednisolone pulse (MP) at 1,000 mg/day for 3 days and followed this with oral administration of prednisolone (PSL) $(40 \mathrm{mg}, 0.6 \mathrm{mg} / \mathrm{kg} /$ day). Following treatment, the corrected visual acuity improved to 0.6 in the right eye and 0.4 in the left eye; SRD and choroidal thickening had ameliorated but still persisted. On the eighteenth day, we administered a second course of steroid semipulse therapy (MP at $500 \mathrm{mg} /$ day for 3 days) and followed this with a slowly tapering course of oral PSL from $40 \mathrm{mg}$ /day to $30 \mathrm{mg} /$ day in 4 weeks. After 2 courses of steroid pulse therapy, followed by oral administration of steroids, the bestcorrected visual acuity improved to 1.2 in the right eye and 1.0 in the left eye, the SRD had disappeared and CT reduced (shown in Fig. 2c, d).

When the dose was gradually reduced to PSL at $10 \mathrm{mg} /$ day, only nivolumab was rechallenged for the first time in 4 months after the drug was discontinued. There was no relapse of uveitis, and the PSL was further reduced to $5 \mathrm{mg} /$ day. After the second dose of nivolumab, EDI-OCT showed choroidal thickening in both eyes (shown in Fig. 2e, f). There were no inflammatory findings other than the rethickening of the choroid. In previous reports, choroidal thickening was indicative of latent choroidal inflammation, which occurs prior to the recurrence of VKH [5, 6]. STTA was immediately administered, and choroidal thickening was resolved as it recovered to the same level as in remission (shown in Fig. 2g, h). At the fourth dose of nivolumab, ipilimumab was added due to the exacerbation of malignant melanoma. Mild iritis appeared, but this was improved by continuing PSL $5 \mathrm{mg} /$ day and adding steroid eye drops. The dose of PSL was increased to $40 \mathrm{mg} /$ day due to liver damage of IRAE, and no recurrence of ICPIU was observed during the 5-month follow-up period. At the last visit, a mild sunset glow fundus appeared. No alopecia or vitiligo was identified.

\section{Discussion}

The frequency of ocular complications due to ICPI is reported to be about $1-3 \%[7,8]$. ICPIU is the most common ocular complication caused by the use of ICPI (15.1\%). Other complications include retinal disorders, optic nerve disorders, and dry eye. ICPIU has been reported to have a higher incidence in melanoma than in lung cancer. Of the ICPIU, VKH disease-like uveitis accounted for about $8 \%$ of cases, of which $68.8 \%$ was related to melanoma [8]. Moreover, no gender or age differences have been reported [9].

$83.6 \%$ of patients with ICPIU develop uveitis within 6 months (median 63 days) after the start of ICPI administration [9], but this case developed as early as 25 days after the combined administration of the 2 drugs. Combination therapy is said to have a higher antitumor effect than single-agent administration, but the incidence of IRAE is also higher [10]. The incidence of uveitis is also highest in the IRAE [8].

Nivolumab, an anti-PD-1 antibody, suppresses the PD-1/PD-L1 pathway, which is thought to enhance the immune response and cause excessive eye inflammation. Since CTLA- 4 is also expressed in normal human melanocytes, ipilimumab, an anti-CTLA-4 antibody, is thought to lead to VKH disease-like uveitis by causing an excessive T-cell response in the choroid containing melanocytes. Combination therapy may enhance the inflammatory response and may make uveitis more likely to develop and become more severe.

VKH is a systemic autoimmune disease targeting melanocytes that affect the eyes, skin, ears, and meninges. The uveitis commonly presents with multiple SRD, hyperemia and edema of the optic nerve head, and thickening of the posterior choroid. Early diagnosis and prompt start of systemic steroid treatment are important [11]. HLA is present in antigen-presenting cells and triggers an immune response by presenting antigen to CD4-positive $\mathrm{T}$ cells. The HLA-DR4 serotype is considered a risk factor [11], and this case was HLA-DR4 positive. Table 1 shows the reports of 6 cases of VKH-like uveitis induced by ICPIs, including cases in

\section{Karger's}




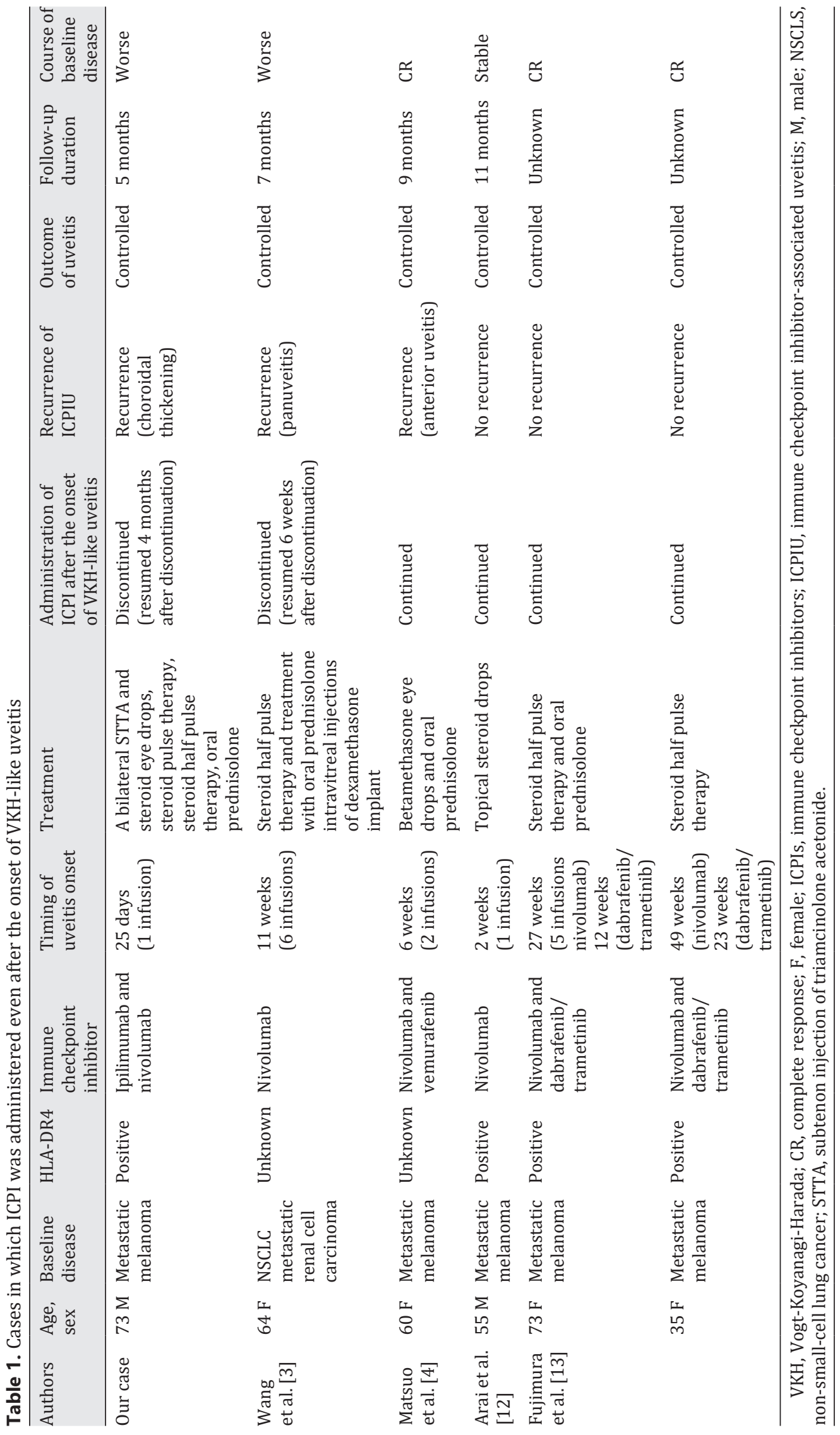


which ICPI was administered even after the onset of VKH-like uveitis [3, 4, 12, 13]. Four cases underwent HLA typing, and all of the cases were HLA-DR4 positive $[12,13]$. All HLA-DR4-positive patients were East Asian (Japanese). Although the incidence of ICPIU is reported to be high in Caucasian (74\%) [9], the incidence of VKH disease-like uveitis induced by ICPI may also be high in East Asian patients; however, further reports are needed for verification.

The treatment protocol for VKH disease-like uveitis induced by ICPIs has not been established. Some cases are treated with only steroid eye drops, and some cases are treated with systemic PSL and discontinuation of ICPIs $[3,4,12,13]$. According to the ICPIU classification of Common Terminology Criteria for Adverse Event, our case was a grade 4, as visual acuity deteriorated to 0.1 or worse. Grade 4 toxicity is recommended to be treated with high-dose systemic steroids and permanent discontinuation of immunotherapy. In this case, moderate iritis, severe SRD, and choroidal thickening were observed from the initial stage. As a result, discontinuation of immunotherapy and high-dose systemic steroid treatment was required to control ocular inflammation. However, due to worsening melanoma, it was necessary to restart immunotherapy.

In cases like ours, continuous use of ICPI administration was required to control the baseline disease even after the onset of ICPIU. However, the number of case reports is small, and no treatment protocol for such cases has been established. Table 1 shows reported cases in which ICPI was administered even after the onset of VKH-like disease. Four cases occurred with continued immunotherapy $[3,4,12,13]$, and 2 cases occurred in which immunotherapy was resumed after discontinuation [3]. No recurrence of ICPIU occurred in 3 of 4 patients who underwent continuous immunotherapy. Conversely, ICPIU recurrence occurred in the 2 patients who resumed immunotherapy, and the baseline disease worsened even after the resumption of immunotherapy. Even in cases where ICPIU was controlled, the risk of recurrence of ICPIU may be high when ICPI administration is resumed. Wang et al. [3] controlled recurrent ICPIU with bilateral intravitreal injections of a dexamethasone implant. In our case, recurrent ICPIU was controlled with oral PSL. Currently, the recurrence of ICPIU is controlled by a combination of various treatments. Attention should be paid to the deterioration of the baseline disease due to the discontinuation of ICPI and recurrence of ICPIU associated with the resumption of immunotherapy. Since the baseline disease is life threatening and the exacerbation of uveitis can cause blindness, treatment strategies should be carefully discussed with each specialist. Further studies are needed to establish the best treatment protocol in the future.

During careful follow-up, EDI-OCT showed choroid thickening. In the treatment of VKH disease, relapse may occur during steroid tapering. During steroid tapering, an increase of $100 \mu \mathrm{m}$ or more from the minimum CT measured by EDI-OCT is considered to be the rebound of CT. The rebound of CT is reported to be an early sign for the recurrence of VKH disease, as an increase in choroidal thickening appears prior to other relapsed inflammatory findings $[5,6]$. In this case, the CT after the second steroid pulse was $390 \mu \mathrm{m}$ in the right eye and 330 $\mu \mathrm{m}$ in the left eye, whereas the CT after readministration of nivolumab was $665 \mu \mathrm{m}$ in the right eye and $485 \mu \mathrm{m}$ in the left eye. No other ocular inflammation findings were observed. In our case, the use of EDI-OCT in follow-up led to early relapse detection. Treatment with STTA reduced the CT and made it possible to continue ICPI. To the best of our knowledge, this is the first report to measure changes in CT to assess relapse of ICPI-induced VKH disease-like uveitis.

We reported a case of VKH disease-like uveitis induced by administration of nivolumab plus ipilimumab in a patient with metastatic melanoma. Although steroid treatment was effective, long-term follow-up is required because uveitis may recur when ICPI administration is restarted. As VKH disease is a vision-threatening disease, it may be necessary to check HLA typing prior to ICPI administration to predict the risk of onset, especially for East

\section{Karger's}


Asian people. When administering ICPI in consideration of the risk of VKH disease-like uveitis, it may be useful to follow-up using EDI-OCT.

\section{Statement of Ethics}

This case report was conducted ethically in accordance with the World Medical Association Declaration of Helsinki. Written and informed consent was obtained for publication from the patient. This study does not include any information which may reveal the patient's identity. This study protocol was reviewed and the need for approval was waived by the Ethics Committee of Tokushima University Hospital.

\section{Conflict of Interest Statement}

The authors have no conflicts of interest to declare.

\section{Funding Sources}

No funding was received.

\section{Author Contributions}

All authors participated in the analysis of this case. All authors reviewed and approved the manuscript.

\section{Data Availability Statement}

All data generated or analyzed during this study are included in this article and its online supplementary material files (see www.karger.com/doi/10.1159/000520416 for all online suppl. material). Further enquiries can be directed to the corresponding author.

\section{References}

1 Pardoll DM. The blockade of immune checkpoints in cancer immunotherapy. Nat Rev Cancer. 2012 Mar 22; 12(4):252-64.

2 Ai M, Curran MA. Immune checkpoint combinations from mouse to man. Cancer Immunol Immunother. 2015 Jul;64(7):885-92.

3 Wang W, Lam WC, Chen L. Recurrent grade 4 panuveitis with serous retinal detachment related to nivolumab treatment in a patient with metastatic renal cell carcinoma. Cancer Immunol Immunother. 2019 Jan;68(1): 85-95.

4 Matsuo T, Yamasaki O. Vogt-Koyanagi-Harada disease-like posterior uveitis in the course of nivolumab (antiPD-1 antibody), interposed by vemurafenib (BRAF inhibitor), for metastatic cutaneous malignant melanoma. Clin Case Rep. 2017 Mar;5(5):694-700.

5 Nakayama M, Keino H, Okada AA, Watanabe T, Taki W, Inoue M, et al. Enhanced depth imaging optical coherence tomography of the choroid in Vogt-Koyanagi-Harada disease. Retina. 2012 Nov-Dec;32(10):2061-9.

6 Tagawa Y, Namba K, Mizuuchi K, Takemoto Y, Iwata D, Uno T, et al. Choroidal thickening prior to anterior recurrence in patients with Vogt-Koyanagi-Harada disease. Br J Ophthalmol. 2016 Apr;100(4):473-7.

7 Dalvin LA, Shields CL, Orloff M, Sato T, Shields JA. Checkpoint inhibitor immune therapy: Systemic indications and ophthalmic side effects. Retina. 2018 Jun;38(6):1063-78. 
8 Bomze D, Meirson T, Hasan Ali O, Goldman A, Flatz L, Habot-Wilner Z. Ocular adverse events induced by immune checkpoint inhibitors: a comprehensive pharmacovigilance analysis. Ocul Immunol Inflamm. 2020 Aug; 4:1-7.

9 Dow ER, Yung M, Tsui E. Immune checkpoint inhibitor-associated uveitis: review of treatments and outcomes. Ocul Immunol Inflamm. 2021 Jan;29(1):203-11.

10 Larkin J, Chiarion-Sileni V, Gonzalez R, Grob JJ, Rutkowski P, Lao CD, et al. Five-year survival with combined nivolumab and ipilimumab in advanced melanoma. N Engl J Med. 2019 Oct;381(16):1535-46.

11 Patil YB, Garg R, Rajguru JP, Sirsalmath M, Bevinakatti VA, Kumar M, et al. Vogt-Koyanagi-Harada (VKH) syndrome: a new perspective for healthcare professionals. J Family Med Prim Care. 2020 Jan;9(1):31-5.

12 Arai T, Harada K, Usui Y, Irisawa R, Tsuboi R. Case of acute anterior uveitis and Vogt-Koyanagi-Harada syndrome-like eruptions induced by nivolumab in a melanoma patient. J Dermatol. 2017 Aug;44(8):975-6.

13 Fujimura T, Kambayashi Y, Tanita K, Sato Y, Hidaka T, Otsuka A, et al. HLA-DRB1*04:05 in two cases of VogtKoyanagi-Harada disease-like uveitis developing from an advanced melanoma patient treated by sequential administration of nivolumab and dabrafenib/trametinib therapy. J Dermatol. 2018 Jun;45(6):735-7. 